\title{
Analytical Transmission Scanning Electron Microscopy: Extending the Capabilities of a Conventional SEM Using an Off-the-Shelf Transmission Detector*
}

\author{
Jason Holm ${ }^{1}$ and Robert Keller ${ }^{1}$. \\ 1. National Institute of Standards and Technology, Materials Measurement Lab., Boulder, CO 80305
}

Transmission-mode scanning electron microscopy techniques are seeing increased use in both materials science and biological applications [1]. One reason is that recently available off-the-shelf transmission detectors that can be implemented into nearly any scanning electron microscope (SEM) are enabling imaging modes not normally associated with conventional SEMs. For example, some modular units include bright and dark field transmission detectors, and one modular unit includes a high angle annular dark field (HAADF) detector with which elemental contrast can be observed.

Although modular transmission detectors have demonstrated much utility [1-3], their full capacity has not yet been realized. One drawback is the inability to easily control the detector collection angle, and therefore select which electrons will be used to form images. Unlike scanning transmission electron microscopes (STEMs), conventional SEMs do not have post-specimen projector lenses that can be used to modify the collection angle. This means either (1) the specimen-to-detector distance must be adjusted to change the collection angle, or (2) an appropriate mask/aperture must be used to restrict the scattered electrons to a particular region of the detector corresponding to the desired collection angle. Furthermore, SEM transmission detectors with built-in specimen holders do not allow the sample position or orientation to be changed with respect to the detector or incident electron beam, and commercially available detectors with separate holders allow very little specimen positional control. (For example, see Fig. 1 in [2].) Inserting masks/apertures between the specimen and detector in such cases is challenging since very little space is available, and aligning an aperture with the detector and the optic axis is cumbersome at best.

This contribution demonstrates a method to extend the analytical capacity of existing off-the-shelf modular detectors by implementation of (1) a new sample holder, and (2) a simple, inexpensive aperture system that can be used to select electrons scattered to any angle intercepted by the detector. It will be shown for one particular transmission detector that bright field acceptance angles are not limited to those dictated by the intended bright field detector, and that acceptance angles are easily controllable and modifiable over a wide range, from $0 \mathrm{mrad}$ to $>1350 \mathrm{mrad}$. Implementation of other transmission imaging modes including annular bright field (ABF) [4], low angle annular dark field (LAADF) [5], and thin annular detector (TAD) schemes [6] is also possible. In particular, this work shows how the two developments can enable HAADF transmission imaging in a 15 year old SEM.

To demonstrate the utility of a cantilevered, clamp-based sample holder and aperture system, Figure 1 shows an interior view of the SEM chamber with a transmission detector and EDS detector inserted. An annular aperture (not directly visible in the chamber image) was placed between the transmission detector and the sample. With this aperture in place, and the sample positioned approximately $6 \mathrm{~mm}$ above the detector, the dark field inner acceptance angle was approximately $250 \mathrm{mrad}$. Figure 2 shows dark field images of several $30 \mathrm{~nm}$ diameter $\mathrm{Au}$ and $\mathrm{TiO}_{2}$ nanoparticles on a carbon substrate recorded under these conditions. The image intensity differences for different materials are easily discernible in Fig 2a. Figure $2 \mathrm{~b}$ shows an X-ray map of the sample, demonstrating that the most intense regions can

*Contribution of the U.S. Department of Commerce; not subject to copyright in the United States. 
be assigned to the Au particles. Figure 2c shows that Au particles hidden under an agglomeration of $\mathrm{TiO}_{2}$ particles are not visible when viewed with a conventional secondary electron detector, but are easily discernible when imaging with the transmission detector and annular aperture system.

This work shows that a relatively low cost upgrade can significantly extend the analytical capabilities of practically any SEM [8].

\section{References:}

[1] T. Klein, et al., in Advances in Imaging and Electron Physics, P.W. Hawkes (Ed.), 171 (2012), 297.

[2] N. Hondow et al. Nanotoxicology 5 (2011) 215.

[3] M. Kuwajima et al. PLOS ONE 8 (2013) e59573.

[5] S. D. Findlay et al. Ultramicroscopy 110 (2010) 903.

[6] G. Rubin et al. Ultramicroscopy 113 (2012) 131.

[7] J. M. Cowley, J. Electron Micros. 50 (2001) 147.

[8] J. Holm acknowledges funding from the National Research Council Postdoctoral Research Associateship Program.

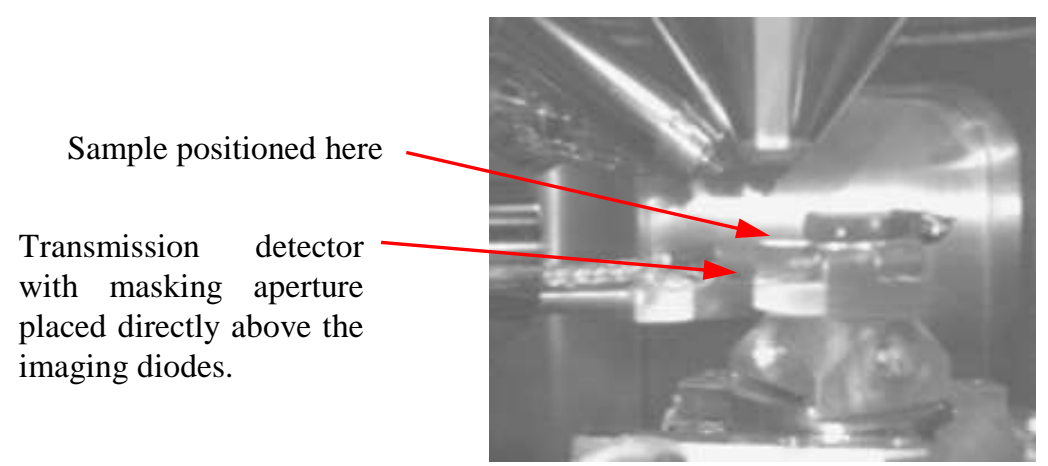

Figure 1. A view of the SEM chamber interior with the transmission detector, sample holder, and EDS detector inserted. This is the setup used to generate the images shown in Fig. 2. Because of the camera angle, the sample and aperture are not visible in this image.

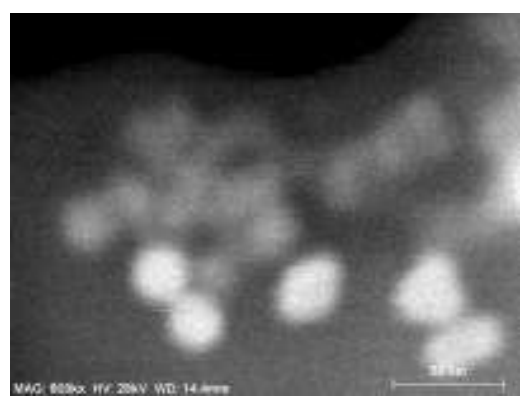

(a)

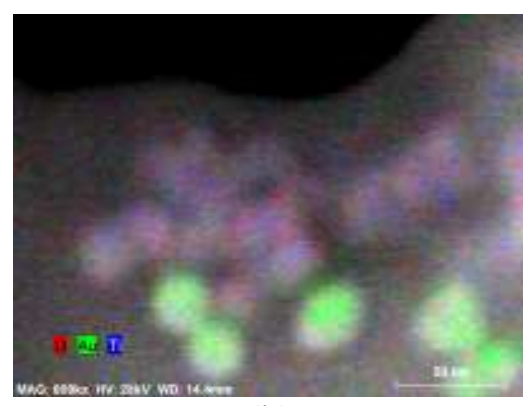

(b)

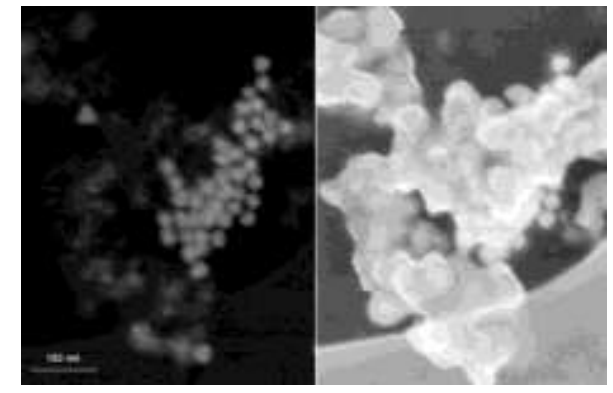

(c)

Figure 2. HAADF transmission images showing $30 \mathrm{~nm}$ Au nanoparticles and $\approx 30 \mathrm{~nm} \mathrm{TiO}_{2}$ nanoparticles on a carbon substrate. The detector gain has been adjusted to show the carbon substrate. (a) An image showing intensity differences between the $\mathrm{Au}, \mathrm{TiO}_{2}$, and $\mathrm{C}$. (b) An X-ray map superimposed on the HAADF image. (c) Split-screen image showing Au particles under a pile of $\mathrm{TiO}_{2}$ particles. (HAADF image is on the left, secondary electron image is on the right.) 